\title{
Correction to: Macleay's Choice: Transacting the Natural History Trade in the Nineteenth Century
}

\author{
Simon Ville ${ }^{1}\left(\mathbb{D} \cdot\right.$ Claire Wright $^{2} \cdot$ Jude Philp $^{3}$
}

Published online: 17 August 2020

(c) Springer Nature B.V. 2020

\section{Correction to: Journal of the History of Biology https://doi.org/10.1007/s10739-020-09610-9}

During the publication process of above mentioned article the Notes to Figs. 1, 2, 3 , and 4 were erroneously deleted from the figure legends. The correct versions are given below:

Fig. 1 Macleay's global network of agents. Note Ties between Sydney and other cities indicates that Macleay had an agent in that city. Thicker ties indicate more agents or more frequent transactions. Scale 1:125,000,000

Fig. 2 Macleay's global network of agents-Asia-Pacific. Note Ties between Sydney and other cities indicates that Macleay had an agent in that city. Thicker ties indicate more agents or more frequent transactions. Scale 1:45,000,000

Fig. 3 Macleay's global network of agents-monetary transactions. Note Ties between Sydney and other cities indicates that Macleay had an agent in that city. Thicker ties indicate more agents or more frequent transactions. Scale 1:125,000,000

Fig. 4 Macleay's global network of agents-exchange transactions. Note Ties between Sydney and other cities indicates that Macleay had an agent in that city. Thicker ties indicate more agents or more frequent transactions. Scale 1:125,000,000

The original article has been corrected.

The original article can be found online at https://doi.org/10.1007/s10739-020-09610-9.

Simon Ville

sville@uow.edu.au

1 University of Wollongong, Wollongong, Australia

2 Macquarie University, Sydney, Australia

3 University of Sydney, Sydney, Australia 
Publisher's Note Springer Nature remains neutral with regard to jurisdictional claims in published maps and institutional affiliations. 